\title{
Manejo endoscópico de papiloma invertido nasosinusal. Experiencia de 15 años en Hospital Clínico Regional de Concepción
}

\author{
Endoscopic management of sinonasal inverted papilloma. \\ 15 years experience in Concepción Clinical Hospital
}

\author{
Alfredo Santamaría C', Mario Tapia C'1, Felipe Fredes C', David Astudillo 0², Ignacio Mendoza A².
}

\begin{abstract}
RESUMEN
Introducción: El papiloma invertido es uno de los tumores benignos más frecuentes de la región nasosinusal. Los principales problemas en su manejo son la recurrencia y transformación maligna. Su tratamiento es la extirpación quirúrgica.

objetivos: Describir y analizar las características clínicas y epidemiológicas, así como el resultado del tratamiento endoscópico practicado en el Hospital Clínico Regional de Concepción.

Material y método: Se realizó un estudio retrospectivo, descriptivo, de todos los pacientes con diagnóstico de papiloma invertido nasosinusal operados vía abordaje endoscópico entre los años 1997 y 2012. Se analizaron variables epidemiológicas y clínicas, incluyendo análisis de las recurrencias y transformación/asociación a cáncer.

Resultados: Se encontraron 57 casos de papiloma invertido nasosinusal, 55 de los cuales se abordaron endoscópicamente, 65,5\% hombres, 54,7 años en promedio de edad. La obstrucción nasal fue el principal síntoma. La mayor cantidad se concentró en estadio 2 (47,3\%) y $3(32,7 \%)$ de Krouse. Complicaciones relevantes 7,8\%, las cuales fueron resueltas sin secuelas. Seguimiento promedio de 4,1 años, con una recurrencia de 5 (9,1\%) casos, una persistencia (1,8\%). Asociación con carcinoma escamoso en el 5,5\% de los casos.

Conclusión: La experiencia con la resección endoscópica en el papiloma invertido nasosinusal ha demostrado ser efectiva en nuestro medio, con baja tasa de complicaciones y recurrencias.
\end{abstract}

Palabras clave: Cirugía endoscópica nasal, papiloma invertido nasosinusal, tumor nasosinusal, papiloma schneideriano.

\section{ABSTRACT}

Introduction: The inverted papilloma is one of the most common benign tumors of the sinonasal region. The main problems in their management are the recurrence and malignant transformation. Their treatment is surgical removal.

1 Médico Servicio de Otorrinolaringología y Cirugía de Cabeza y Cuello, HCRC.

2 Médico Servicio de Otorrinolaringología, Hospital Erminda Martín, Chillán. 
Aim: To describe and analyze the clinical and epidemiological characteristics, as well as the results of endoscopic treatment in a tertiary hospital.

Materials and methods: A retrospective, descriptive study of all patients with diagnosis of sinonasal inverted papilloma operated by endoscopic approach between 1997 and 2012 was carried out. Epidemiological and clinical variables were analyzed, including analysis of recurrences and transformation to / association with cancer.

Results: 57 cases of sinonasal inverted papilloma were found, 55 of which were endoscopically resected, $65.5 \%$ male, 54.7 years old on average. Nasal obstruction was the main symptom. Most of them were in Krouse stage 2 (47.3\%) and 3 (32.7\%); $7.8 \%$ of major complications, which were resolved without sequels. The follow up average was 4.11 years, with a recurrence of $5(9.1 \%)$ cases, and (1.8\%) persistence. Association with squamous-cell carcinoma was found in $5.5 \%$ of cases.

Conclusion: The clinical and epidemiological characteristics do not differ greatly from those reported in the literature. Experience with endoscopic resection in sinonasal inverted papilloma has proven effective in our clinical practice, with a low rate of complications and recurrences, this approach currently being the first choice in most cases.

Key words: Endoscopic sinus surgery, inverted papilloma, sinonasal tumor, Schneiderian papilloma.

\section{INTRODUCCIÓN}

El papiloma invertido, también conocido como papiloma nasosinusal, epitelial o transicional, es un tumor benigno del epitelio respiratorio nasosinusal, bien diferenciado y localmente agresivo, descrito por primera vez en 1854 por Ward 1 . En 1935, Ringertz acuñó el término "papiloma invertido" cuando observó epitelio invirtiéndose dentro del estroma subyacente $^{2}$. Se caracteriza por un alto riesgo de recurrencia $(12 \%-20 \%)^{3}$ y puede asociarse a malignización $(3 \%-9 \%)^{4-6}$. La transformación maligna se puede presentar hasta en el $15 \%$ de papilomas invertidos, $1,7 \%-5,6 \%$ de los cuales desarrollan un carcinoma en forma sincrónica ${ }^{7}$.

Su etiología es incierta. El virus papiloma humano ha sido encontrado tanto en papiloma invertido como en la mucosa sana inmediatamente adyacente, implicándolo como una posible causa ${ }^{4}$.

Los papilomas invertidos son relativamente raros en la población general, y representan el 0,5\%-4\% de los tumores nasosinusales ${ }^{8}$. Tienen una predominancia por el sexo masculino, con una relación hombre-mujer de 3,4:1, y se presentan principalmente entre la quinta y sexta década, aunque se han visto a cualquier edad 6 .

El papiloma invertido debería ser sospechado en cualquier persona con poliposis unilateral ${ }^{6,9}$, donde el síntoma cardinal es la obstrucción nasal. Otros síntomas incluyen la epistaxis, epífora, cefaleas, rinorrea e hiposmia. El tratamiento quirúrgico es el gold standard, siendo la resección completa de su base de implantación lo más importante para prevenir la recurren$\mathrm{cia}^{10-11}$. Los sitios más frecuentes de origen son el etmoides, pared lateral nasal y el seno maxi$\operatorname{lar}^{6}$. El abordaje endoscópico actualmente se ha convertido en la vía de elección, con resultados tan buenos como la aproximación abierta ${ }^{4}$, posicionándose como el abordaje de elección para el tratamiento de la gran mayoría de los papilomas invertidos ${ }^{4-9}$.

\section{OBJETIVOS}

Describir y analizar las características clínicas y epidemiológicas, así como el resultado del tratamiento endoscópico de los papilomas invertidos practicado en el Hospital Clínico Regional de Concepción.

\section{MATERIAL Y MÉTODO}

Se realizó un estudio de corte retrospectivo, descriptivo, de todos los pacientes con diagnóstico de papiloma invertido nasosinusal operados entre los años 1997 y 2012 en el Hospital Clínico 
Tabla 1. Etapificación de Krouse

\begin{tabular}{|ll|}
\hline T1 & Confinado sólo a fosa nasal \\
T2 & Complejo osteomeatal y etmoides c/s pared medial maxilar c/s fosa nasal \\
T3 & Resto de paredes de seno maxilar y/o frontal c/s etmoides c/s fosa nasal \\
T4 & Compromiso de órbita, endocráneo, espacio pterigomaxilar y/o malignidad. \\
\hline
\end{tabular}

Regional de Concepción por cualquier vía en forma consecutiva. Se analizaron variables epidemiológicas como edad, sexo y antecedentes mórbidos, así como variables clínicas, incluyendo sintomatología, tiempo de duración de los síntomas antes de la primera consulta, hallazgos al examen físico y en tomografía computarizada.

Todos los casos fueron clasificados según la etapificación propuesta por Krouse $^{12}$ la cual se describe en la Tabla 1.

Se hizo un especial análisis de las recurrencias en cuanto a la búsqueda de causas y relación con la cirugía practicada, así como de los casos de transformación/asociación con cáncer.

\section{RESULTADOS}

Se encontraron 57 casos de papiloma invertido en total, de los cuales 55 fueron operados por vía endoscópica y correspondieron a la población estudiada.

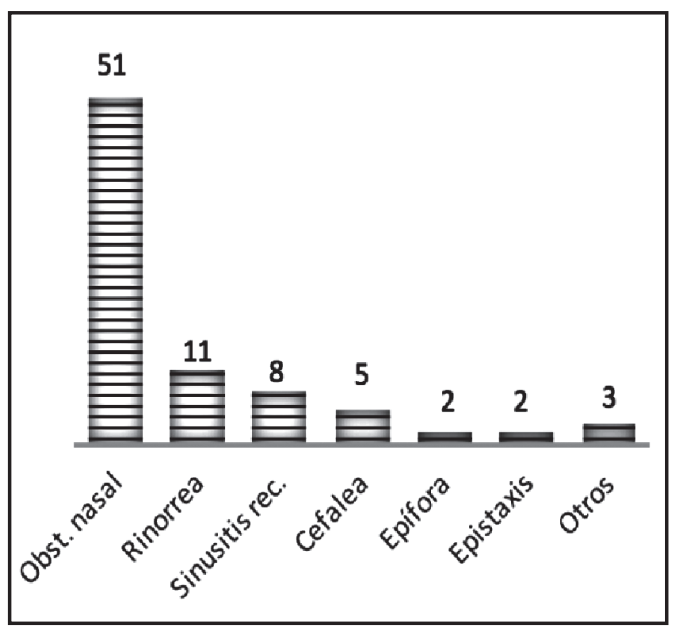

Figura 1. Molestias asociadas a papiloma invertido (n).
La relación hombre:mujer fue de 1,9:1, con una edad promedio en años de 53,9 (9-83). Cinco pacientes habían sido intervenidos previamente, cuatro por papiloma invertido vía abierta y uno por poliposis vía endoscópica.

El síntoma más frecuentemente descrito en el $92,7 \%$ de los casos fue obstrucción nasal. La Figura 1 muestra el resto de la sintomatología registrada en las historias clínicas.

El tiempo de evolución desde el inicio de los síntomas hasta la primera consulta por especialista fue en el $65,5 \%$ superior a un año, mientras que el $14,5 \%$ fue menor a seis meses.

El tumor se presentó bilateral en 5 pacientes $(9,09 \%)$, izquierdo en 16 pacientes $(29,1 \%)$, derecho en 31 pacientes $(56,3 \%)$, sin obstrucción en 1 caso (1,8\%), y en 2 casos no se especificó en ficha clínica. En la Figura 2 se puede observar la etapificación de los casos según la clasificación de Krouse.

En 46 casos $(83,6 \%)$ se identificó papiloma invertido exclusivo, en 6 pacientes $(10,9 \%)$ se

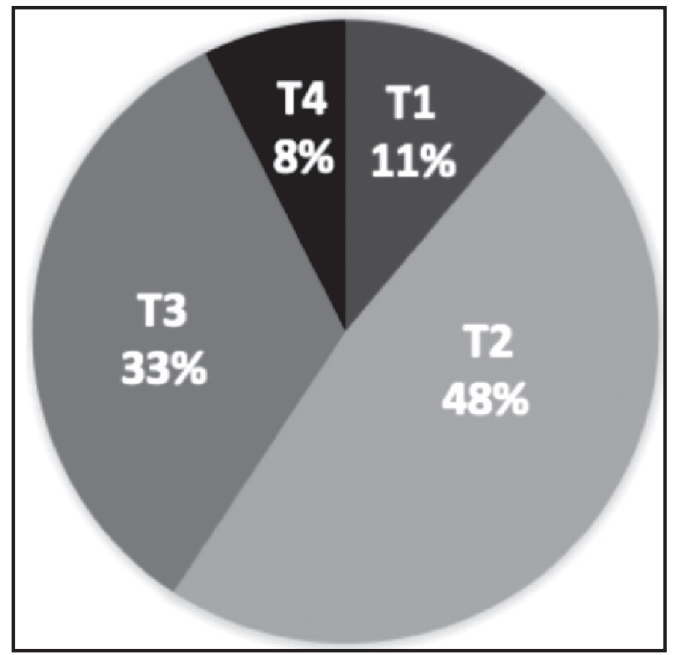

Figura 2. Etapificación clínica según clasificación Krouse. 
asoció a pólipos y en 3 casos (5,5\%) se asoció sincrónicamente a carcinoma escamoso.

La resección del tumor fue endoscópica en todos los casos. En el período revisado no se encontraron abordajes combinados ni abiertos. Hubo 4 complicaciones posoperatorias, 2 obstrucciones de vía lagrimal y 2 fístulas de líquido cefalorraquídeo; el promedio de días de hospitalización fue de 2,87 días (1-9).

El tiempo de seguimiento promedio fue de 48 meses (3-120), encontrándose 5 recidivas (9,1\%), 3 en pared anterior de seno maxilar, 1 en vía lagrimal y la última en el techo del seno esfenoidal. Hubo un caso de persistencia $(1,8 \%)$, asociado a cáncer escamoso.

Finalmente, de los 3 casos asociados a cáncer escamoso, 2 fueron hombres, con una edad promedio de 60,6 años, y fueron tratados exclusivamente con radioterapia posoperatoria una vez hecho el diagnóstico anatomopatológico. Hasta la finalización de este estudio, y luego de 36 meses de seguimiento promedio, no se ha producido recidiva de estos 3 casos.

\section{DISCUSIÓN}

El papiloma invertido nasosinusal es una neoplasia benigna que puede ser diagnosticada a cualquier edad, predominando en la quinta y sexta décadas, especialmente en hombres ${ }^{6}$. Lo anterior es coincidente con nuestro promedio de edad de 53,9 años y $65,5 \%$ de hombres. En relación a la clínica encontrada en los pacientes analizados no se encontraron diferencias con lo reportado en la literatura, clínica que es inespecífica, que se confunde muchas veces con patología rinosinusal crónica y que retrasa por ello la consulta al especialista, en nuestro caso la mayoría demoró más de 1 año $(65,5 \%)$.

En nuestro servicio se solicita de forma rutinaria TAC de senos paranasales a estos pacientes, sin embargo, un parte de ellos no se encontró disponible en el sistema, ya sea por la antigüedad del caso o por la posibilidad de haberse realizado el estudio fuera del hospital. Lo anterior es una limitación de nuestro trabajo.

En lo concerniente a la etapificación según Krouse, en la literatura se pueden encontrar di- ferentes series de casos donde la mayoría se concentran en etapas 2 y 3 , lo cual también es concordante con nuestros resultados ${ }^{13-15}$, con una predominancia en etapa 2 en este estudio (48\%). La asociación con cáncer escamoso se presentó en $5,5 \%$ del total de pacientes, cifra levemente por debajo de las series publicadas, pero que pudiera explicarse por el tamaño reducido de la mues$\operatorname{tra}^{4,5,10,15}$. Estos casos correspondieron a diagnósticos entregados por la biopsia posoperatoria, siendo manejados todos con radioterapia.

En relación al tratamiento, el desarrollo de la cirugía endoscópica ha entregado una serie de ventajas en comparación con el abordaje externo, tales como una mejor visualización, magnificación y detalle del campo operatorio. Permite una mejor determinación de tejido viable no comprometido por el tumor, causando menos costras, menos sangrado y menos dolor posoperatorio. De esta manera, los abordajes tradicionales del pasado han ido perdiendo su utilidad como tratamiento de elección $n^{4,6,7,9,14,15}$.

El porcentaje de recurrencia en nuestro estudio fue de $9,1 \%$. Lo anterior es un factor importante a la hora de elegir un abordaje, y en relación a esto, dos revisiones sistemáticas de varias series de resección de papiloma invertido, demostraron una recurrencia de $12 \%$ para los abordajes endoscópicos versus $17 \%-20 \%$ para los abordajes externos $^{16,17}$. De todas formas, independiente del abordaje, la resección completa y el fresado de la zona de inserción permitirían disminuir las recurrencias ${ }^{2,19}$. Una revisión sistemática de papilomas invertidos de seno frontal demostró una recurrencia de $22,4 \%{ }^{18}$, mientras que en otro estudio hubo $16,9 \%$ de recurrencia para tumores alojados en el seno maxilar ${ }^{19}$. En ambos casos la dificultad en el acceso al seno frontal y a la pared lateral/anterior del seno maxilar es la mejor explicación para estos porcentajes. En nuestra serie, 3 recurrencias fueron en la pared anterior de seno maxilar, pese a haber realizado maxilectomía medial en todos los casos, 1 en vía lagrimal y 1 en techo de seno esfenoidal. En este último caso, el protocolo operatorio describió que impresionaba el origen del papiloma invertido en tabique esfenoidal.

A pesar de no ser una gran casuística el buen resultado obtenido, es similar o mejor a otras experiencias publicadas. Lo anterior probablemente 
se debe a la experiencia en cirugía endoscópica de los cirujanos y a la aplicación de una técnica resectiva agresiva.

\section{BIBLIOGRAFÍA}

1. WaRD N. A mirror of the practice of medicine and surgery in the hospitals of London: London hospital. Lancet 1854; 2: 480-2.

2. Anari S, Carrie S. Sinonasal inverted papilloma: narrative review. J Laryngol Otol 2010; 124: 705-15.

3. VRABEC DP. The inverted Schneiderian papilloma: a 25-year study. Laryngoscope 1994; 104: 582-605.

4. Lund VJ, Stammberger H, Nicolal P, et al. European position paper on endoscopic management of tumours of the nose, paranasal sinuses and skull base. Rhinol Supp/2010; 1-143.

5. СноI J, Kim S, KIm Y, et AL. Clinical and histologic features of inverted papilloma-associated malignancy. Eur Arch Otorhinolaryngol 2012; 269: 2349-54.

6. Lawson W, Patel Z. The evolution of management for inverted papilloma: an analysis of 200 cases. Otolaryngol Head Neck Surg 2009; 140: 330-5.

7. Peng $P, H_{A R}-E l$ G. Management of inverted papillomas of the nose and paranasasinuses. Am J Otolaryngol 2006; 27: 233-7.

8. Mirza S, Bradley PJ, Acharya A, et al. Sinonasal inverted papillomas: recurrence, and synchronous and metachronous malignancy. J Laryngol Otol 2007; 121: 857-64.

9. Lombardi D, Tomenzol D, Butta L, et al. Limitations and complications of endoscopic surgery for treatment for sinonasal inverted papilloma: a reassessment after 212 cases. Head Neck 2011; 33: 1154-61.
10. Lawson W, Kaufman MR, Biller HF. Treatment outcomes in the management of inverted papilloma: an analysis of 160 cases. Laryngoscope 2003; 113: 1548-56.

11. Lawson W, Ho BT, Shaari CM, Biller HF. Inverted papilloma: a report of 112 cases. Laryngoscope 1995; 105: 282-8.

12. Krouse JH. Development of a staging system for inverted papilloma. Laryngoscoope 2000; 110(6): 965-8.

13. Tomenzoli D, Castelnuovo P, Pagella F, Berlucchi M, Pianta L, Delù G, et al. Different endoscopic surgical strategies in the management of inverted papilloma of sinonasal tract: experience with 47 patients. Laryngoscope 2004; 114(2): 193200.

14. Souza LA, Verde RCL, Lessa MM, Lessa HA, LIMA CMF. Endoscopic treatment of sinonasal papilloma: a retrospective clinical study. Int Arch Otorhinolaryngol 2010; 14(3): 351-4.

15. KIm DY, Hong SL, LeE CH. Inverted papilloma of the nasal cavity and paranasal sinuses: a Korean multicenter study. Laryngoscope 2012; 122(3): 487-94.

16. Karkos PD, Fyrmpas G, Carrie SC, Swift aC. Endoscopic versus open surgical interventions for inverted nasal papilloma: a systematic review. Clin Otolaryngol 2006; 31: 499-503.

17. Busquets JM, Hwang PH. Endoscopic resection of sinonasal inverted papilloma: a meta-analysis. Otolaryngol Head Neck Surg 2006; 134: 47682.

18. Walgama E, Ahn C, Batra PS. Surgical management of frontal sinus inverted papilloma. Laryngoscope 2012; 122: 1205-9.

19. Healy DY JR, ChHabra N. Surgical risk factors for recurrence of inverted papilloma. Laryngoscope 2015.

\author{
Dirección: Alfredo Santamaría C. \\ Servicio de Otorrinolaringología y Cirugía de Cabeza y Cuello \\ Hospital Clínico Regional de Concepción \\ San Martín 1436 \\ E mail: asantamaria@vtr.net
}

